\title{
Reversible Conversion in the Brassinosteroid Quartet Castasterone, Brassinolide and their $3 \beta$-Epimers
}

Andrey P. Antonchick ${ }^{\mathrm{a}, \mathrm{b}}$, Aleš Svatoš ${ }^{\mathrm{a}}$, Olga V. Konstantinova ${ }^{\mathrm{b}}$, Vladimir N. Zhabinskii ${ }^{\mathrm{b}}$, Vladimir A. Khripach ${ }^{\mathrm{b}}$, and Bernd Schneider ${ }^{\mathrm{a}}$

${ }^{a}$ Max-Planck-Institute for Chemical Ecology, Beutenberg-Campus, Hans-Knöll-Str. 8, D-07745 Jena, Germany

${ }^{b}$ Institute of Bioorganic Chemistry, National Academy of Sciences of Belarus, Kuprevich Str., 5/2, 220141 Minsk, Belarus

Reprint requests to Dr. B. Schneider. Fax: +49-3641-571601. E-mail: schneider@ice.mpg.de

Z. Naturforsch. 61b, 1039 - 1044 (2006); received January 31, 2006

The metabolism of deuterated brassinosteroids has been studied in excised leaves of Secale cereale, and in vitro in seedlings of Arabidopsis thaliana and cell suspension cultures of Lycopersicon esculentum. In addition to the known biosynthetic conversion of castasterone to brassinolide and epimerization to 3 -epicastasterone, inversion of the $3 \alpha$-configured hydroxyl group of brassinolide to a $3 \beta$-configured one in 3 -epibrassinolide has been observed using liquid chromatography (HPLC) with electrospray ionization (ESI) and selected ion-monitoring mass-spectrometry (SIM-MS). Administration of deuterated 3-epicastasterone and 3-epibrassinolide to Arabidopsis and Secale seedlings resulted in the formation of castasterone and brassinolide, respectively, indicating conversion of configuration at C-3 of brassinosteroids is reversible.

Key words: Biosynthesis, Brassinosteroids, Epimerization

\section{Introduction}

The biosynthesis and metabolism of brassinosteroids in plants has been studied by means of labelling experiments and mutant studies, and on the enzymatic level $[1-5]$. Clearly brassinosteroides are a part of a complex regulatory system controlling plant homeostasis $[6,7]$. An important precondition of its correct functioning is maintenance of a proper level of this hormone, which can be achieved by combining the corresponding biosynthetic (activation) and metabolic (inactivation) reactions. Recent studies have provided increasing evidence for a cross-linked biosynthetic grid of interchangeable steps rather than a distinct route to brassinolide [8-10] as well as strong evidence for deactivation of brassinosteroids in plants. The last may be the reason why results of experiments on the practical use of brassinolide (the most potent phytohormone among brassinosteroids) have been disappointing [11] Evidently, the level of this endogenous hormone is strictly regulated in plants, whereas inactivation mechanisms of its analogs may be less efficient. This could be the reason why brassinolide analogs are more effective in field trials [12]. An excess of brassinolide and other brassinosteroids can be removed (or stored) in various ways including hydroxylation/degradation of the side chain and conjugation with fatty acids, sugars or sulfate [12-16] or inactivated by unknown mechanisms [17]. The present work deals with the possibility that transformations in ring $\mathrm{A}$ are involved in brassinolide-level control.

Inversion of configuration of the hydroxy group at C-3 represents one of the reactions, which can occur at different steps in the pathway. Inversion from $3 \beta$ to $3 \alpha$ configuration has been demonstrated in the course of early and late C-6 oxidation [18 - 19], and 3epi-6-deoxocathasterone ( $3 \alpha$-configuration) was identified as a metabolite of 6-deoxocathasterone $(3 \beta$ configuration) [9]. Formation of teasterone (3 $\beta$ epimer) from typhasterol ( $3 \alpha$-epimer) through the 3 dehydro intermediate showed the reversibility of this reaction [20]. Brassinosteroids of the $24 \beta$ series have also been used to show that inversion of configuration at C-3 is influenced by regio- and stereospecific formation of carbohydrate conjugates of the $3 \beta$ epimer [21]. Compared to $3 \beta$-isomers, $3 \alpha$-isomers generally are more active in the rice lamina inclination bioassay. The conversion of $3 \beta$ to $3 \alpha$ is supposed to represent an activation step, and the opposite, $3 \alpha$ to $3 \beta$, e.g. castasterone to 3 -epicastasterone [22], is con- 
sidered an inactivation step. Using 24-epicastasterone and 24-epibrassinolide, transformation to $3 \beta$-isomers was shown by cell suspension cultures of Ornithopus sativus [23]. Thus, inversion of configuration at C-3 is one of the putative possibilities to inactivate brassinolide, the most active brassinosteroid, but this specific reaction has not yet been experimentally demonstrated. The reverse conversion of 3-epicastasterone and 2epicastasterone to castasterone was detected recently in seedlings of Secale cereale [10]. The conversion of brassinolide to 3-epibrassinolide, a naturally occurring brassinosteroid in Arabidopsis [24], and the reverse conversion are reported here for the first time and, together with the corresponding conversions between castasterone and 3-epicastasterone, are discussed in the context of hormone homeostasis in plants.

\section{Results and Discussion}

Brassinosteroids in Arabidopsis thaliana and Secale cereale

The occurrence of the brassinosteroids castasterone (1), brassinolide (2), 3-epicastasterone (3) and 3epibrassinolide (4), which are subject to the present metabolic study, was searched in the literature and investigated in Arabidopsis thaliana and Secale cereale. In $A$. thaliana, brassinosteroids [25-26] have been reported several times [27-29]. Castasterone (1) and brassinolide (2) are native also to Arabidopsis shoots and seeds, and 3-epibrassinolide (4) was recently found in root-callus suspension cultures of ecotype C24 [24]. Schmidt et al. [30] reported castasterone (1) together with other brassinosteroids from seeds of S. cereale. Recently we demonstrated the presence of castasterone (1) and 3-epicastasterone (3) in seeds of this plant [10].

Here we investigate the occurrence of brassinosteroids in Arabidopsis thaliana seedlings grown under in vitro conditions. The seedlings were extracted according to the procedure described in Materials and Methods. Triply deuterated brassinosteroids, $\left[26-{ }^{2} \mathrm{H}_{3}\right] 3$-epicastasterone, and $\left[26-{ }^{2} \mathrm{H}_{3}\right] 3-$ epibrassinolide [31] $(0.4 \mu \mathrm{g}$ in $10 \mu \mathrm{l}$ of $75 \% \mathrm{EtOH}$ each), were added as internal standards. Several solvent partitioning and chromatographic steps resulted in brassinosteroid-containing fractions, which after derivatization with an excess of dansyl-3aminophenylboronic acid were analyzed by liquid chromatography (HPLC) with electrospray ionization (ESI) and using an established selected ion-monitoring
Table 1. Detection of brassinosteroids by relative retention times of their 22,23-dansyl-3-aminophenylboronates. SIM channels were recorded at given $\mathrm{m} / \mathrm{z}$ values. Conditions: Macherey-Nagel Nucleodur 100-3 C18 column $(100 \mathrm{~mm} \times$ $1 \mathrm{~mm}, 3 \mu \mathrm{m})$; gradient $\mathrm{MeCN}-0.1 \% \mathrm{HCOOH}$ in $\mathrm{H}_{2} \mathrm{O}(0-$ 10 min: $75 \% \mathrm{MeCN} ; 10-15$ min: $75-100 \% \mathrm{MeCN} ; 15-$

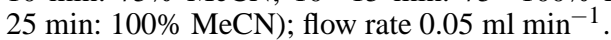

\begin{tabular}{lcccc}
\hline & $R R_{\mathrm{t}}{ }^{*}$ & $\begin{array}{c}m / z \\
{[\mathrm{M}+\mathrm{H}]^{+}}\end{array}$ & $\begin{array}{c}m / z \\
\left.\left[{ }^{2} \mathrm{H}_{3}\right] \mathrm{M}+\mathrm{H}\right]^{+}\end{array}$ & $\begin{array}{c}m / z \\
\left.\left[{ }^{2} \mathrm{H}_{6}\right] \mathrm{M}+\mathrm{H}\right]^{+}\end{array}$ \\
\hline Castasterone (1) & 1.419 & 799 & 802 & 805 \\
Brassinolide (2) & 1.000 & 815 & 818 & 821 \\
3-Epicastasterone (3) & 1.100 & 799 & 802 & 805 \\
3-Epibrassinolide (4) & 0.857 & 815 & 818 & 821 \\
\hline
\end{tabular}

* Relative retention time with respect to brassinolide $(\mathbf{1})(R=$ 11.15 min).

mass-spectrometric (SIM-MS) detection (HPLC-ESISIM-MS) method [10,32]. Identification of brassinosteroids based on the relative retention time of 22,23-dansyl-3-aminophenylboronates (Table 1). Using this approach, castasterone (1), brassinolide (2), 3-epicastasterone (3) and 3-epibrassinolide (4) were detected in Arabidopsis seedlings. The relative retention times of authentic standards and mass spectral data (Table 1) also revealed the presence of castasterone (1) and 3-epicastasterone (3) in seedlings of $S$. cereale.

\section{Metabolism of castasterone and brassinolide}

$\left[26,28-{ }^{2} \mathrm{H}_{6}\right]$ Castasterone (1) and $\left[26,28-{ }^{2} \mathrm{H}_{6}\right]$ brassinolide (2) [33] were administered in a series of separate experiments in different plant materials, seedlings of A. thaliana $S$. cereale, and cell suspension cultures of L. esculentum. After a two-day incubation, the plant material was extracted according to the procedure used to detect endogenous brassinosteroids. Brassinosteroid-containing fractions were converted to their dansyl-3-aminophenylboronates and analyzed by HPLC-ESI-SIM-MS [10, 32].

Administering $\left[26,28-{ }^{2} \mathrm{H}_{6}\right]$ castasterone (1) to $A$. thaliana and L. esculentum and analysing the extracts by HPLC-ESI-SIM-MS, we identified sixfold deuterated brassinolide $(2)\left(\mathrm{m} / \mathrm{z} 821 ;[\mathrm{M}+\mathrm{H}]^{+} ; R R_{\mathrm{t}}=1.000\right)$ in both plants. The conversion of castasterone (1) to brassinolide (2) (Fig. 1) confirmed that this well established final step of brassinolide biosynthesis is operating in our experimental system. SIM analysis for the molecular mass of sixfold deuterated castasterone (1) $\mathrm{m} / \mathrm{z} 805\left([\mathrm{M}+\mathrm{H}]^{+}\right)$allowed us to identify the parent compound 1 at $R R_{\mathrm{t}} 1.419$ and 3-epicastasterone (3) at $R R_{\mathrm{t}} 1.100$ of the same molecular mass. The natural abundance isotopomer of $\mathbf{3}$, the $3 \beta$-epimer 


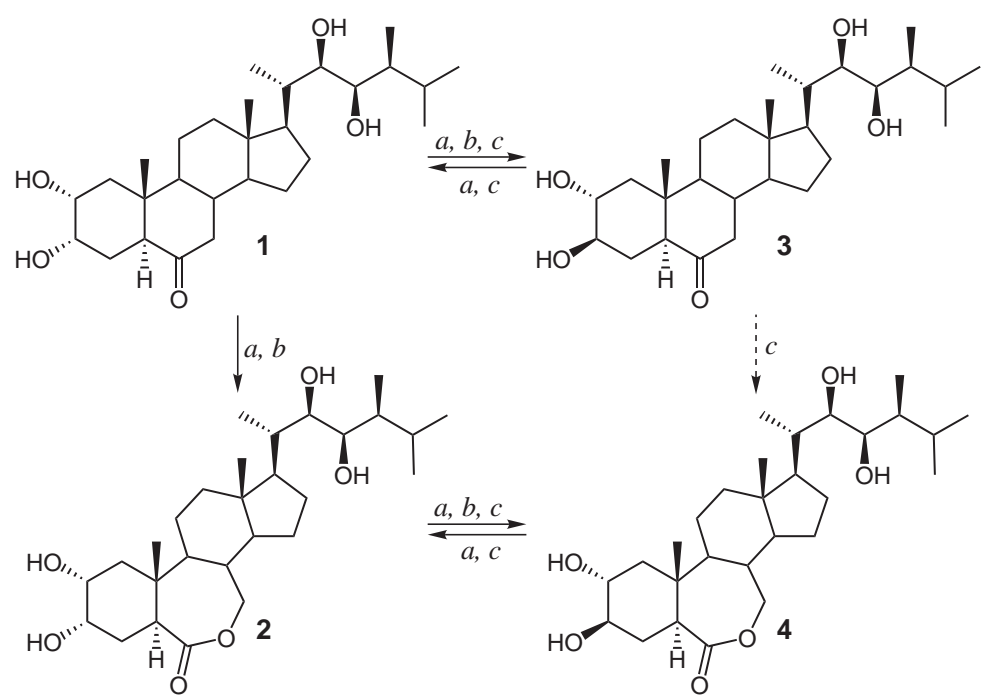

Fig. 1. Conversion of brassinosteroids by in vitro-grown seedlings of $A$. thaliana (a), cell suspension cultures of L. esculentum $(b)$, and seedlings of $S$. cereale (c). Dashed arrow: Putative conversion. of $1\left(\mathrm{~m} / \mathrm{z}\right.$ 799; $\left.[\mathrm{M}+\mathrm{H}]^{+} ; R R_{\mathrm{t}} 1.101\right)$, was detected in seedlings of $A$. thaliana as well. Furthermore, in seedlings of $S$. cereale $\left[26,28-{ }^{2} \mathrm{H}_{6}\right]$ castasterone (1) was converted to labelled 3-epicastasterone $(\mathbf{3})(\mathrm{m} / \mathrm{z}$ 805; $[\mathrm{M}+\mathrm{H}]^{+}$) as the only product (Fig. 1). The discovery of compound $\mathbf{3}$ as a metabolite of $\mathbf{1}$ resembles previous results [22], where in the seedlings of Catharanthus roseus the conversion of $\mathbf{1}$ to $\mathbf{2}$ and $\mathbf{3}$ was observed, while the conversion of $\mathbf{1}$ to only $\mathbf{3}$ was found in the seedlings of Nicotiana tabacum and Oryza sativa.

Administering $\left[26,28-{ }^{2} \mathrm{H}_{6}\right]$ brassinolide (2) followed by an analogous separation procedure resulted in identification of sixfold labelled 3-epibrassinolide (4) $\left(\mathrm{m} / \mathrm{z}\right.$ 821; $\left.[\mathrm{M}+\mathrm{H}]^{+} ; R R_{\mathrm{t}} 0.857\right)$ in A. thaliana, $S$. cereale seedlings, and tomato cell suspension (Fig. 1). The metabolic conversion of brassinolide, the most active plant steroid hormone, to its $3 \beta$-epimer is considered an inactivation process. This result is the first experimental evidence for metabolic inactivation of brassinolide (1) in plants by stereochemical alteration. However, inversion of configuration at C-3 has been already observed for 24-epibrassinolide in cell cultures of Ornithopus sativus [34].

Metabolism of 3-epicastasterone and 3-epibrassinolide

The previously reported reversibility of the epimerization of the 3-hydroxyl group of brassinosteroids $[20,21,35,36]$ encouraged us to investigate the fate of 3-epicastasterone (3) and 3-epibrassinolide (4) in seedlings of A. thaliana and $S$. cereale.
In order to check for the conversion of $\left[26-{ }^{2} \mathrm{H}_{3}\right] 3-$ epicastasterone (3) and 3-epibrassinolide (4) to $3 \alpha$ hydroxybrassinosteroids, both deuterated compounds $\mathbf{3}$ and $\mathbf{4}$ were administered in separate experiments. Screening the analogously prepared extract from feeding $\left[26-{ }^{2} \mathrm{H}_{3}\right] 3$-epicastasterone (3) for the mass of triply deuterated 1 and its isomers $\left(\mathrm{m} / \mathrm{z}, 802,[\mathrm{M}+\mathrm{H}]^{+}\right)$ showed a peak corresponding to labelled precursor $\mathbf{3}$ $\left(R R_{\mathrm{t}} 1.100\right)$ and a peak corresponding to labelled castasterone $1\left(R R_{\mathrm{t}} 1.419\right)$ in the seedlings of $A$. thaliana and $S$. cereale. In addition a peak corresponding to triply deuterated 3-epibrassinolide (4) $(\mathrm{m} / \mathrm{z}$ 818; $\left.[\mathrm{M}+\mathrm{H}]^{+} ; R R_{\mathrm{t}} 0.856\right)$ in the seedlings of $S$. cereale has been detected (Fig. 1). This conversion is the first evidence that 3-epicastasterone (3) is an alternative biosynthetic precursor of 3-epibrassinolide (4). However, additional studies with substrates specifically deuterated at $\mathrm{C}-2$ and $\mathrm{C}-3$ are necessary to check for the course $\mathbf{3} \rightarrow \mathbf{1} \rightarrow \mathbf{2} \rightarrow \mathbf{4}$ including so far undetected direct lactonization of $\mathbf{1}$ to $\mathbf{2}$ and hypothetical conversion of 3-dehydrocastasterone to 3dehydrobrassinolide in this plant.

In analogous experiments, $\left[26-{ }^{2} \mathrm{H}_{3}\right] 3$-epibrassinolide (4) was administered to the seedlings of $A$. thaliana and $S$. cereale. HPLC-ESI-SIM-MS analysis showed triply labelled brassinolide $(2)\left(\mathrm{m} / z 818 ;[\mathrm{M}+\mathrm{H}]^{+} ; R R_{\mathrm{t}}\right.$ $0.856)$ in extracts of plants treated with the $3 \beta$-epimer 4 (Fig. 1). These results showed that the conversion of $3 \beta$ - to $3 \alpha$-epimers is operating in A. thaliana and $S$. cereale not only for 6-oxo but 7-homolactonetype brassinosteroids also. As demonstrated by the identifica- 
tion of $3 \beta$-epimers as metabolites of $3 \alpha$-stereoisomers and vice versa, $3 \alpha$-epimers as metabolites of $3 \beta$ stereoisomers, the inversion of configuration is possible in both directions. If $3 \alpha \rightarrow 3 \beta$ inversion of configuration represents inactivation, the reverse conversion must be considered as activation. However, 3epibrassinolide (4) may be not simply an inactive metabolite but a potential precursor of brassinolide (2) which, under certain conditions, could be reconstituted if required for homeostasis of the plant cell. Knowledge of these processes could be used for developing compounds with prolonged brassinosteroid activity for agricultural and horticultural application.

Several 2-epibrassinosteroids such as 2-epicastasterone, 2-epi-25-methyldolichosterone and 2-epi-23dehydrobrassinolide $[10,37,38]$ and conversions of 2epicastasterone to castasterone and 24-epibrassinolide to 2,24-diepibrassinolide are known [10,39]. Given the results of the present investigation, reversibility of the conversion of stereochemistry at C-2 may be analogous to conversions of stereochemistry at C-3, a possibility which should be investigated.

\section{Experimental Section}

Plant materials and administration of labelled precursors

Cultures of A. thaliana cv. C24 were freshly established from surface-sterilized seeds for each experiment. Seeds were germinated and grown in $100 \mathrm{ml}$ conical flasks containing liquid auxin- and cytokinin-free B5 medium $(30 \mathrm{ml})$ [40] containing sucrose $\left(20 \mathrm{~g} \mathrm{l}^{-1}\right)$. During germination and growth the flasks were maintained at $23{ }^{\circ} \mathrm{C}$ on a rotary shaker at $85 \mathrm{rpm}$ under constant illumination $\left(15 \mu \mathrm{mol} \mathrm{m} \mathrm{m}^{-2} \mathrm{~s}^{-1}\right)$. After a culture period of 3 to 4 weeks, the seedlings reached a fresh weight of approximately $15 \mathrm{~g}$ and were used at that stage for feeding experiments. A solution of the respective deuterated precursor in $75 \% \mu \mathrm{l} \mathrm{EtOH}(20 \mu \mathrm{l})$ was added to the medium under sterile conditions and incubated for two days. Each of the deuterated precursors $\left[26,27-{ }^{2} \mathrm{H}_{6}\right]$ castasterone (1), $\left[26,27-{ }^{2} \mathrm{H}_{6}\right]$ brassinolide (2), $\left[26-{ }^{2} \mathrm{H}_{3}\right] 3$-epicastasterone (3), [26- $\left.{ }^{2} \mathrm{H}_{3}\right] 3$-epibrassinolide (4) (0.4 to $4 \mu \mathrm{g}$ per flask) was administered individually. Three to four flasks were used in parallel experiments.

Seeds of S. cereale cv. "Sorom" were purchased from Lochow-Petkus GmbH, Bergen-Wohlde, Germany. Prior to germination, seeds were stratificized and then grown in the greenhouse at $22-24{ }^{\circ} \mathrm{C}$ using a mixture of Vermiculite and sand (ratio $3: 1$ ) as a substrate. The natural photoperiod was supplemented with $16 \mathrm{~h}$ illumination from Philips Sun-T Agro $400 \mathrm{Na}$ lights. Biosynthetic experiments were performed using freshly harvested leaves of 2- to 3-week-old seedlings of $S$. cereale cv. "Sorom" (30 to $60 \mathrm{~g}$ ). $\left[26,27-{ }^{2} \mathrm{H}_{6}\right]$ Castasterone (1), $\left[26,27-{ }^{2} \mathrm{H}_{6}\right]$ brassinolide (2), $\left[26-{ }^{2} \mathrm{H}_{3}\right] 3$-epicastasterone $(3),\left[26-{ }^{2} \mathrm{H}_{3}\right] 3-$ epibrassinolide (4) were administered separately to plant material. In typical experiments, the respective deuterated precursor $(0.2$ to $10 \mu \mathrm{g})$ was dissolved in $75 \% \mathrm{EtOH}(10 \mu \mathrm{l})$ and added to a flask containing freshly harvested leaves immersed in water $(30 \mathrm{ml})$. The precursor was absorbed hydroponically with the transpiration stream and incubated for $70 \mathrm{~h}$, during which time water was periodically added to the feeding solution.

Cell suspension cultures of L. esculentum L. cv. "Tamina" were grown in Linsmaier-Skoog medium [41] in $300 \mathrm{ml}$ conical flasks containing $100 \mathrm{ml}$ cell suspension at $23{ }^{\circ} \mathrm{C}$ on a rotary shaker at $85 \mathrm{rpm}$ under constant diffuse light $\left(4.4 \mu \mathrm{mol} \mathrm{m}^{-2} \mathrm{~s}^{-1}\right)$. Subculturing was performed every $14 \mathrm{~d}$ using an inoculum of approximately $40 \mathrm{ml}$. In typical experiments, a filter-sterilized solution of the respective deuterated precursor (1 to $20 \mu \mathrm{g}$ ) in $75 \%$ ethanol $(20 \mu \mathrm{l})$ was added to flasks with plant material ( 55 to $60 \mathrm{~g}$ ) and incubated for three days. Each of the deuterated precursors $\mathbf{1}$ and $\mathbf{2}$ was administered individually.

\section{Identification of endogenous brassinosteroids in Arabi- dopsis}

The seedlings of $A$. thaliana cv. C24 were separated from the medium and extracted with $\mathrm{MeOH}(3 \times 80 \mathrm{ml})$ using an Ultra-Turrax homogenizer. $\left[26-{ }^{2} \mathrm{H}_{3}\right] 3$-Epicastasterone (3), and $\left[26-{ }^{2} \mathrm{H}_{3}\right] 3$-epibrassinolide (4) [31] $(0.4 \mu \mathrm{g}$ in $10 \mu \mathrm{l}$ of $75 \% \mathrm{EtOH}$ each) were added as internal standards before evaporation followed by the procedure described below. Identification of endogenous brassinosteroids $\mathbf{1}$ to $\mathbf{4}$ was based on a comparison of retention times and relative intensities of molecular ions.

\section{Isolation and analysis of brassinosteroid metabolites}

After incubation with labelled precursors, the plant materials were separated from the medium and extracted with $\mathrm{MeOH}(3 \times 80 \mathrm{ml})$ using an Ultra-Turrax homogenizer. The aqueous residue remaining from evaporation of the methanolic extract was partitioned between equal volumes of EtOAc and $0.5 \mathrm{M} \mathrm{K}_{2} \mathrm{HPO}_{4}(3 \times 100 \mathrm{ml})$. The culture medium was extracted with EtOAc $(100 \mathrm{ml})$ and the EtOAc phase was combined with the corresponding extract obtained from the plant material. The combined EtOAc phase was evaporated; $\mathrm{MeOH}(10 \mathrm{ml})$ was added, sonified for $15 \mathrm{~min}$, and centrifuged for $15 \mathrm{~min}$ at $4000 \mathrm{rpm}$ at $15^{\circ} \mathrm{C}$. The supernatant was evaporated and subjected to TLC (silica gel $60 \mathrm{~F}_{254}, 0.5 \mathrm{~mm}$ thickness, $200 \times 200 \mathrm{~mm}$, Merck; $\mathrm{CHCl}_{3}$ $\mathrm{MeOH} 88: 12$ ). Based on the retention times of authentic standards, which were determined on a separate plate (compound 1: $R_{\mathrm{f}} 0.41 ; 2$ and $3: R_{\mathrm{f}} 0.31 ; 4: R_{\mathrm{f}} 0.25$ ), the 
brassinosteroid-containing zone $\left(R_{\mathrm{f}} 0.21-0.45\right)$ was collected and eluted sequentially with a mixture of $\mathrm{CHCl}_{3}-$ $\mathrm{MeOH} 1: 1(20 \mathrm{ml})$ and $\mathrm{MeOH}(30 \mathrm{ml})$. The combined solution was evaporated to dryness and subjected to reversedphase HPLC (LiChrospher ${ }^{\circledR} 100$ RP-18; $10 \mu \mathrm{m} ; 250 \times$ $10 \mathrm{~mm}$ ) using a linear gradient $\mathrm{MeCN}-0.01 \%$ trifluoracetic acid in $\mathrm{H}_{2} \mathrm{O}$ from 30 to $100 \% \mathrm{MeCN}$ in 30 min and held at $100 \% \mathrm{MeCN}$ for a further $15 \mathrm{~min}$ (flow rate $2 \mathrm{ml} \mathrm{min}^{-1}$; UV detection of matrix compounds at $205 \mathrm{~nm}$ ). Based on the retention times of authentic standards (compound 1: $R_{\mathrm{t}}$ $\left.23.0 \mathrm{~min} ; 2: R_{\mathrm{t}} 20.9 \mathrm{~min} ; 3: R_{\mathrm{t}} 21.3 \mathrm{~min} ; \mathbf{4}: R_{\mathrm{t}} 19.1 \mathrm{~min}\right)$, the brassinosteroid-containing fraction $\left(R_{\mathrm{t}} 17.5-24.5 \mathrm{~min}\right)$ was collected and evaporated. The residue was converted to bisdansyl-3-aminophenylboronates by being heated to $62^{\circ} \mathrm{C}$ for 30 min with a solution of dansyl-3-aminophenylboronic acid $\left(3 \mathrm{mg} \mathrm{ml}^{-1}\right)$ in a mixture of pyridine and acetonitrile $(1: 19)$ and used for analysis by HPLC-ESI-SIM-MS as previously described [10,32].

\section{Acknowledgements}

We thank Dr. A. Kolbe and Prof. G. Adam (Halle) for providing $\left[26,28-{ }^{2} \mathrm{H}_{6}\right]$ castasterone and $\left[26,28-{ }^{2} \mathrm{H}_{6}\right]$ brassinolide and Emily Wheeler (Jena) for linguistic help in the preparation of this manuscript.
[1] A. Sakurai, in A. Sakurai, T. Yokota, S. D. Clouse (eds): Brassinosteroids. Steroidal Plant Hormones, p. 91 - 111, Springer, Tokyo (1999).

[2] T. Yokota, in P. J. J. Hooykaas, M. A. Hall, K. R. Libbenga (eds): Biochemistry and Molecular Biology of Plant Hormones, p. 277 -293, Elsevier, Amsterdam (1999).

[3] B. Schneider, in K. Esser, U. Lüttge, W. Beyschlag, F. Hellwig (eds): Progress in Botany, Vol. 63, p. 286306, Springer, Berlin (2002).

[4] S. Fujioka, T. Yokota, Ann. Rev. Plant Biol. 54, 137 (2003).

[5] T. Nomura, T. Kushiro, T. Yokota, Y. Kamiya, G. J. Bishop, S. Yamaguchi, J. Biol. Chem. 280, 17873 (2005).

[6] S. Bancos, T. Nomura, T. Sato, G. Molnar, G. J. Bishop, C. Koncz, T. Yokota, F. Nagy, M. Szekeres, Plant Physiol. 130, 504 (2002).

[7] G. T. Kim, H. Tsukaya, J. Plant Res. 115, 169 (2002).

[8] Y. Shimada, S. Fujioka, N. Miyauchi, M. Kushiro, S. Takatsuto, T. Nomura, T. Yokota, Y. Kamiya, G. J. Bishop, S. Yoshida, Plant Physiol. 126, 770 (2001).

[9] S. Fujioka, S. Takatsuto, S. Yoshida, Plant Physiol. 130, 930 (2002).

[10] A. P. Antonchick, A. Svatoš, B. Schneider, O. V. Konstantinova, V.N. Zhabinskii, V. A. Khripach, Phytochemistry 66, 65 (2005).

[11] Y. Kamuro, S. Takatsuto, in H. G. Cutler, T. Yokota, G. Adam (eds): Brassinosteroids: Chemistry, Bioactivity and Applications, p. 292-297, Am. Chem. Soc., Washington, DC (1991).

[12] V.A. Khripach, V. N. Zhabinskii, A. de Groot, Brassinosteroids. A New Class of Plant Hormones, p. 65 -72, Academic Press, San Diego (1999).

[13] H. Suzuki, S. K. Kim, N. Takahashi, T. Yokota, Phytochemistry 33, 1361 (1993).

[14] G. Adam, B. Schneider, in A. Sakurai, T. Yokota, S. D. Clouse (eds): Brassinosteroids. Steroidal Plant Hormones, p. 113-136, Springer, Tokyo (1999).
[15] M. Rouleau, F. Marsolais, M. Richard, L. Nicolle, B. Voigt, G. Adam, L. Varin, J. Biol. Chem. 274, 20925 (1999).

[16] M. M. Neff, S. M. Nguyen, E. J. Malancharuvil, S. Fujioka, T. Noguchi, H. Seto, M. Tsubuki, T. Honda, S. Takatsuto, S. Yoshida, J. Chory, Proc. Natl. Acad. Sci. USA 96, 15316 (1999).

[17] E. M. Turk, S. Fujioka, H. Seto, Y. Shimada, S. Takatsuto, S. Yoshida, H. C. Wang, Q. I. Torres, J. M. Ward, G. Murthy, J. Y. Zhang, J. C. Walker, M. M. Neff, Plant J. 42, 23 (2005).

[18] H. Suzuki, S. Fujioka, S. Takatsuto, T. Yokota, N. Murofushi, A. Sakurai, J. Plant Growth Regul. 13, 21 (1994).

[19] Y. H. Choi, S. Fujioka, T. Nomura, A. Harada, T. Yokota, S. Takatsuto, A. Sakurai, Phytochemistry 44, 609 (1997).

[20] T. Noguchi, S. Fujioka, S. Choe, S. Takatsuto, F.E. Tax, S. Yoshida, K. A. Feldman, Plant Physiol. 124, 201 (2000).

[21] A. Kolbe, B. Schneider, A. Porzel, G. Adam, Phytochemistry 48, 467 (1998).

[22] H. Suzuki, S. Fujioka, S. Takatsuto, T. Yokota, N. Murofushi, A. Sakurai, Biosci. Biotech. Biochem. 59, 168 (1995).

[23] A. Kolbe, B. Schneider, A. Porzel, G. Adam, Phytochemistry 41, 163 (1996).

[24] O. V. Konstantinova, A.P. Antonchick, N. J. Oldham, V. N. Zhabinskii, V. A. Khripach, B. Schneider, Collect. Czech. Chem. Commun. 66, 1729 (2001).

[25] G. Adam, J. Schmidt, B. Schneider, in W. Herz, H. Falk, G.W. Kirby, R.E. Moore, C. Tamm (eds): Progr. Chem. Org. Nat. Prod., Vol. 78, p. 1-46, Springer, Vienna (1999).

[26] A. Bajguz, A. Tretyn, Phytochemistry 62, 1027 (2003).

[27] J. Schmidt, T. Altmann, G. Adam, Phytochemistry 45, 1325 (1997).

[28] S. Fujioka, T. Noguchi, T. Yokota, S. Takatsuto, S. Yoshida, Phytochemistry 48, 595 (1998). 
[29] Y. Shimada, H. Goda, A. Nakamura, S. Takatsuto, S. Fujioka, S. Yoshida, Plant Physiol. 131, 287 (2003).

[30] J. Schmidt, B. Spengler, T. Yokota, M. Nakayama, S. Takatsuto, B. Voigt, G. Adam, Phytochemistry 38, 1095 (1995)

[31] V.A. Khripach, V.N. Zhabinskii, O. V. Konstantinova, A. P. Antonchick, B. Schneider, Steroids 67, 587 (2002).

[32] A. Svatoš, A. P. Antonchick, B. Schneider, Rapid Commun. Mass Sp. 18, 816 (2004).

[33] A. Kolbe, A. Porzel, J. Schmidt, G. Adam, J. Label. Comp. Radiopharm. 46, 231 (2003).

[34] A. Kolbe, B. Schneider, A. Porzel, J. Schmidt, G. Adam, Phytochemistry 38, 633 (1995).

[35] J. Winter, B. Schneider, S. Meyenburg, D. Strack, G. Adam, Phytochemistry 51, 237 (1999).
[36] U. Stündl, B. Schneider, Phytochemistry 58, 989 (2001).

[37] S. K. Kim, in H. G. Cutler, T. Yokota, G. Adam (eds): Brassinosteroids: Chemistry, Bioactivity and Applications. p. 26-35, Am. Chem. Soc., Washington, DC (1991).

[38] T. Watanabe, T. Yokota, K. Shibata, T. Nomura, H. Seto, S. Takatsuto, J. Chem. Res. 18 (2000).

[39] N. Nishikawa, H. Abe, M. Natsume, A. Shida, S. Toyama, J. Plant Physiol. 147, 294 (1995).

[40] O. L. Gamborg, R. A. Miller, K. Ojima, Exp. Cell Res. 50, 151 (1968).

[41] E. M. Linsmaier, F. Skoog, Physiol. Plant. 18, 100 (1965). 